\title{
Guido Rasi
}

In mid-November last year, Guido Rasi began his 5-year mandate as Executive Director of the European Medicines Agency (EMA). Trained as a physician, Rasi has nearly two decades of experience overseeing either research organizations or basic research, including the development of novel drug delivery technologies and preclinical models of carcinoma. More recently, he was Director General of Italy's Italian Medicines Agency for 3 years, during which time he was also a member of the EMA's Management Board. Now, as Executive Director of the European regulatory body, his key goal is to ensure that new pharmacovigilance legislation is implemented to best benefit both drug developers and patients, he told Asher Mullard.

Q What are your goals as head of the EMA? My goals align with my challenges, and one of my key challenges will be the implementation of new pharmacovigilance legislation. This will be both a short-term challenge, in regards to ensuring that we comply with the requirements of the law, but also a longer-term challenge, in that we don't want to miss the opportunities that this legislation offers for assessing the real-life benefits of a medicine. Better monitoring of real-life usage data - when patients with concomitant diseases and treatments take the drug - can give us information both on effectiveness and on safety. And although it might show us unexpected adverse effects, it might also unveil unexpected benefits. The history of medicine teaches us that observation is as important as the original trial.

The first deadline for this challenge is in July, which is when companies need to fill in a set of information for their products and when the Pharmacovigilance Risk Assessment Committee will start meeting. I expect initially the new system will require more work both for regulators and for industry, but after it has started and we have developed our routines I think it will enable an easier way of handling risk management.

I think that we also have to really focus on how to enhance and improve post-licensing activities. There will always be some uncertainty about a drug's safety and efficacy at its time of approval, but there is a lot of room for improvement in the post-marketing arena, both in terms of additional post-marketing requirements and in terms of recovering information about recently approved drugs better and faster.
Q What are your priorities in terms of fostering regulatory science? I think the EMA is pretty much on target in terms of regulatory science. Overall, however, we still need to decrease the level of uncertainty around drug approvals, and I think that benefit-risk methodologies could contribute to this. These methodologies rank different events and data, and as the ranking system is explicit they enable increased predictability and a lower margin for interpretation of findings. They might allow us to move away from making decisions by voting, which sometimes provides unpredictable results. These tools have already been tested in a few agencies around Europe, and the results are very encouraging. A broader pilot project will be launched soon.

\section{Q Increasingly, sponsors also face} uncertainty over reimbursement after drugs are approved. How do you plan to work with payers to enable access to new drugs? One thing we are doing is working with health technology assessment (HTA) bodies to streamline our processes. A first step, that has already been done and that has yielded positive results, is that we now work with HTA bodies to provide joint scientific advice. And requests from industry for such joint advice are increasing. The earlier we decide what the requirements are from us for benefit-risk assessment and from the payers, the easier it will be for investors and drug developers.

Q Regulators and drug developers in the United States are talking about how to enable shorter drug development programmes for

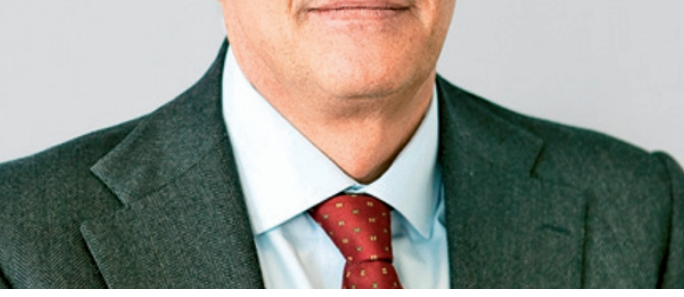

more drugs. Are you thinking about implementing similar schemes in the European Union?

We already have conditional approval pathways in Europe but we are nevertheless collaborating with the US Food and Drug Administration and others on developing new approval approaches as well. The question will be how to implement them.

Two things that have to go with this also may be a bit of a challenge for the industry. First, we would need to be able to apply certain restrictions around prescribing the drugs that are approved via any new pathway because otherwise we are only lowering the evidence standard. Second, we have to be able to ensure that the treatment experience of real-world patients treated under any approval schemes will contribute to future benefit-risk assessments.

\section{Q Industry is keenly awaiting biosimilar guidance documents that are due to be published later this year. What other influential guidance do you expect to issue? There will be guidance on the evaluation of treatments for bacterial infections that will be introduced in January 2013. And we will also issue a lot of guidance documents over the implementation of the pharmacovigilance legislation.}

\section{Q What role do you see for yourself in} encouraging European drug development? We understand that the health of the European drug development sector is not that great at the moment, and we understand that we are competing with other regions. We see ourselves as an enabling agency, and so we do quite a bit to encourage drug development for public health reasons. We think that the scientific advice we give supports the drug development endeavour, as does our commitment to methodological agreements and our work with HTAs.

Overall, my aim is to foster the development of new drugs without compromising the standards and robustness of decision-making. 